\title{
The split Hopkinson bar bulge setup: a novel dynamic biaxial test method
}

\author{
Luca Corallo $^{1 *}$, and Patricia Verleysen ${ }^{1}$ \\ ${ }^{1}$ Department of Electromechanical, Systems and Metal Engineering, MST-DyMaLab Research Group, Ghent \\ University, Technologiepark 46, 9052 Zwijnaarde, Belgium
}

\begin{abstract}
In sheet metal forming, very often, large plastic deformations are imposed to a thin plate. An accurate description of the material's elastoplastic response is therefore of paramount importance to perform finite element (FE) simulations of an actual forming operation. Reliable stressstrain data till significantly larger strains compared to tensile tests can be identified by means of bulge test. In this work, a dynamic hydraulic bulge test is proposed. The novel split Hopkinson bar bulge setup, combines features of classical split Hopkinson pressure bar (SHPB) and hydraulic bulge tests. The special configuration of the Hopkinson bars leaves the sample surface fully accessible. As such, high-speed optical measurements can be performed on the sample surface allowing the application of, for instance, digital image correlation (DIC) for full-field displacement strain mapping. The potential of the facility is explored by performing experiments on $0.8 \mathrm{~mm}$ thick Al2024-T3 sheet.
\end{abstract}

\section{Introduction}

In traditional sheet metal forming processes, a thin sheet metal is deformed at relatively slow speeds. However, more and more the speed of production processes is increased, very often to take advantage of the beneficial implications that high-strain rates can have on the formability of certain metals [1]. The elastoplastic characterization of sheet metals at high strain rates is the first and foremost requirement to correctly design high-speed forming operations during which the material undergoes large plastic deformations. To reach larger uniform strains compared to uniaxial tests, biaxial tensile testing is commonly employed [2]. A few researchers [3], [4] performed quasi-equibiaxial tests at high strain rates by combining a conventional split Hopkinson pressure bar (SHPB) setup with a bulge test apparatus. In the setup realized by Grolleau et al. [3], the input bar is inserted inside a movable hydraulic chamber which holds a circular specimen, while the output bar is put in tight contact with the hydraulic chamber. The bulge pressure is calculated from strain gage measurements on the output bar. Both the bars are made of nylon. The authors proposed a theoretical analysis to describe the plastic deformation of the sheet metal during the experiment, since the sample is not visible during the test. Ramezani et al. [4] presented a variation of the Grolleau setup by introducing a thick polyurethane rubber pad as a medium to transfer the pressure wave

\footnotetext{
* Corresponding author: Luca.Corallo@UGent.be
} 
from the input bar to the sheet metal. The major shortcoming of the bars, bulge cell and sample clamping assembly is that the sample surface is not visible during the entire experiment. Hence, the setups do not allow continuous monitoring of the displacement and strain fields on the sample surface. In the present work, a novel split Hopkinson bar bulge setup, equipped for stereo digital image correlation (DIC) measurements, is presented. The setup combines features of both SHPB and hydraulic bulge tests. Main innovation of the novel facility is the special configuration of the bars which leaves the sample surface accessible for full-field displacement and strain mapping. Thus, the employment of theoretical assumptions and approximations aiming at describing the stress and strain evolutions during the test is not necessary. In this work, the potential of the facility is explored by performing experiments on Al2024-T3 sheet for two different impactor velocities.

\section{Theoretical background}

\subsection{Bulge test}

In bulge experiments, a thin circular plate of initial thickness $t_{0}$ is clamped around its edge to a circular die of diameter $2 a$. The specimen is deformed into a quasi-spherical cap with a polar height $h$ by a hydrostatic pressure $p$ acting at one side of the specimen surface. Assuming an isotropic material, and thus axisymmetric deformation, the membrane stress at the sample apex is described by equation [5]:

$$
\sigma=\frac{p R}{2 t}
$$

Where $R$ is the radius of curvature of the deformed sample and $t$ is the actual thickness. Assuming a material behaviour independent of the hydrostatic pressure, the stress state at the sample apex is equivalent to uniaxial compression acting perpendicularly to the sample surface. The corresponding true strain is derived as:

$$
\varepsilon=\ln \left(\frac{t}{t_{0}}\right)
$$

Eqs. (1), (2) correspond to the von Mises stress and the equivalent strain at the sample apex.

\subsection{Split Hopkinson pressure bar}

In a classical SHPB setup a small sample is sandwiched between two long bars, called respectively input and output bar. A cylindrical impactor, accelerated towards the free end the input bar, generates a compressive incident wave traveling along the input bar towards the sample. The interaction of the compressive pulse with the sample generates a reflected wave which travels back in the input bar and a transmitted wave propagating in the output bar. Strain gage measurements on the two elastic bars are used to measure the incident, reflected and transmitted strain waves indicated with $\varepsilon_{i}, \varepsilon_{r}$ and $\varepsilon_{t}$ respectively. The force $\mathrm{F}_{s}$ acting on the sample is derived by the one-dimensional wave propagating theory as [6]:

$$
F_{s}(t)=A_{\text {out }} \sigma_{t}(t)=A_{\text {out }} E_{\text {out }} \varepsilon_{t}(t)
$$

$\sigma_{t}$ is the transmitted stress wave on the output bar, and $\mathrm{E}_{\text {out }}$ and $\mathrm{A}_{\text {out }}$ are the elastic modulus and output bar section respectively. 


\section{The split Hopkinson bulge setup}

The main innovative feature of the split Hopkinson bulge setup reported in Fig. 1a, compared to existing setups [3], [4], originates from the special configuration of the Hopkinson bars which leaves the sample surface fully accessible for high-speed camera monitoring during the entire experiment. The 6 meters long input bar is concentrically placed inside a 3 meters long coaxial hollow tube used as output bar. The clamping system, shown in detail in Fig. $\mathbf{1 b}$, consists of a holder and die connected at the end of the output bar. The input bar is in contact with a piston, inserted into a hydraulic chamber realized inside the hollow output tube. The impact of the projectile at the free end of the input bar generates a compressive wave traveling in the input bar towards the hydraulic piston. The piston subsequently pressurizes the liquid in the hydraulic chamber and thus deforms the sample. The dynamic load needed to deform the sample is transferred to the output tube as a tensile wave. In the input bar, a reflected tensile wave is observed.

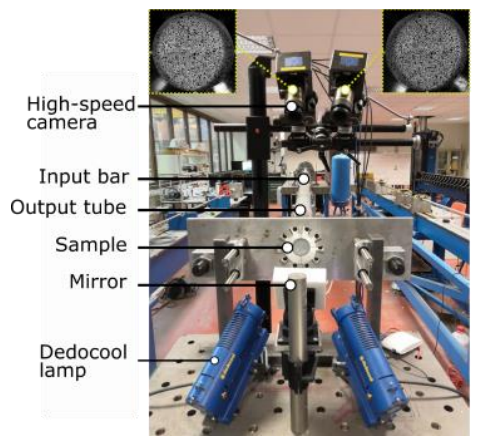

(a)

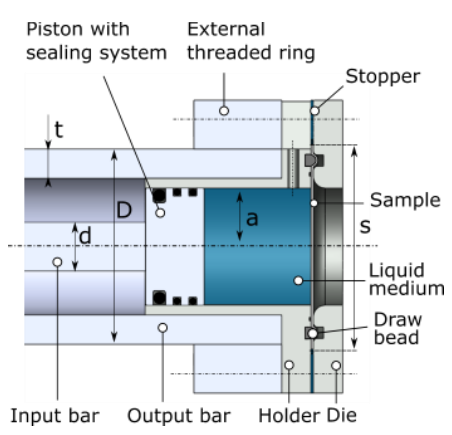

(b)

Fig. 1. Split Hopkinson bar bulge setup installed at Ghent University (a) and schematic representation components hydraulic chamber (b) $(d=25 \mathrm{~mm}, D=100 \mathrm{~mm}, t=15 \mathrm{~mm}, a=30 \mathrm{~mm}$, $\mathrm{s}=52 \mathrm{~mm}$ )

Eq. (3) is adapted to derive the pressure acting at the sample surface:

$$
p(t)=\frac{A_{\text {out }} E_{\text {out }}}{\pi a^{2}} \varepsilon_{t}(t)
$$

Both input and output bars are made of Al7075-T6 because of its high yield strength, i.e., $570 \mathrm{MPa}$, and relatively low Young's modulus, i.e., $72 \mathrm{GPa}$, which allows to impose a highmagnitude compressive waves, and at the same time to maximize the deformation capacity of the setup. All the components of the hydraulic chamber, except for the steel stopper, are made of high-grade aluminum to reduce the weight of the system, and thus the effect of the inertia. The maximum duration of an experiment is approximately $1.2 \mathrm{~ms}$.

\section{Experimental procedure}

High-speed bulge experiments are performed on 0.8mm thick Al2024-T3 sheet. Al2024 is widely employed in the forming industry because of its good machinability, relatively high ultimate tensile strength, and low density. Before an experiment, a stochastic white/black speckle pattern is applied to the sample surface. The sample surface is continuously recorded during an experiment using two high-speed cameras. The cameras used are Photron mini AX200 cameras equipped with two Tamron lenses of 90mm focal length. The cameras are 
used together with a mirror with high surface quality placed in front of the sample while its surface is illuminated by two Dedocool lamps (see Fig. 1a). From the recorded images, fullfield displacement and strain fields are extracted using the 3D commercial DIC software provided by MatchID. A framerate of $30000 \mathrm{fps}$ is chosen giving images with a spatial resolution of $512 \times 384$ pixels. The order of magnitude of the resolution is of about $10^{-3} \mathrm{~mm}$ and $10^{-4}$ for the displacement and strain fields respectively. The procedure used to determine the flow curve of the tested material is based on the ISO16808:2014 standard. Before an experiment, water is introduced into the hydraulic chamber and the input bar is placed in contact with the piston.

\section{Results and discussion}

\subsection{Strain gage signals}

Fig. 2 shows the strain signals shifted to the sample interfaces for two different impactor velocities 9 and $16 \mathrm{~m} / \mathrm{s}$. The transmitted waves are amplified by a factor of 5 for clarity. After an initial rise time of about $80 \mu$ s, relatively constant strain amplitudes, of $1320 \mu$ strain, and $1850 \mu$ strain are achieved. The mismatch of mechanical impedance at the input bar/piston interface generates a wave reflection which is observed at the beginning of the reflected wave. Since the reflection is propagating away from the sample, it does not affect the sample deformation. For the experiment at $9 \mathrm{~m} / \mathrm{s}$, the drop in the transmitted wave corresponds with the end of loading since fracture does not occur. In the experiment at $16.5 \mathrm{~m} / \mathrm{s}$, on the contrary, the sample reaches the fracture during testing. For this test, the sharp drop indicates the sample fracture. In Fig. 3 the forces acting at the liquid/sample and output bar/sample interfaces are plotted together. The good agreement between the forces acting on the two bars fulfils the force equilibrium condition required during Hopkinson experiments.

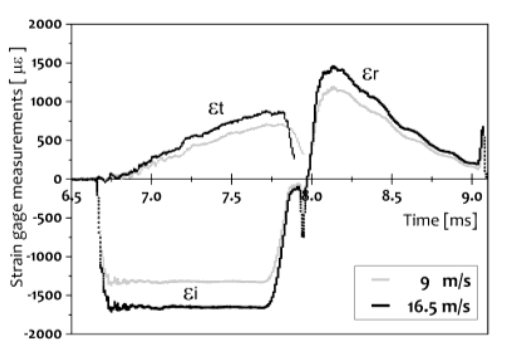

Fig. 2. Shifted strain signals for two different impactor velocities

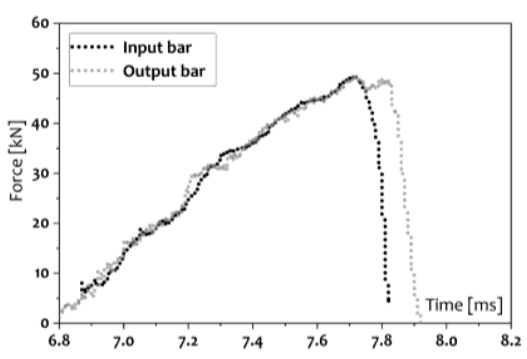

Fig. 3. Force equilibrium input and output bar for the experiment at $16.5 \mathrm{~m} / \mathrm{s}$

The apparent shorter duration of the force signal recorded in the input bar is related to the interference between the incident wave and its reflection at the free end of the input bar. It should be noted that a nearly oscillation-free transmitted wave is obtained, which guarantees a steady sample loading.

\subsection{DIC results}

The contour plots of the rolling and transverse strains, at difference stages of the experiment at $16.5 \mathrm{~m} / \mathrm{s}$, are compiled in Fig. 4. Differences between the two strains are observed in terms of strain amplitudes and distribution. The highest strain values are found at the sample apex. No undesirable concentration of deformation occurs at the sample clamping. The fracture 
starts at the apex and propagates along the rolling direction. The strain fields, during the last stages of the experiment (7.8-7.9ms), exhibit an elliptical distribution. Indeed, the strain in the rolling direction has a higher intensity along the transverse direction. On the contrary, the strain in the transverse direction is higher along the rolling axis.

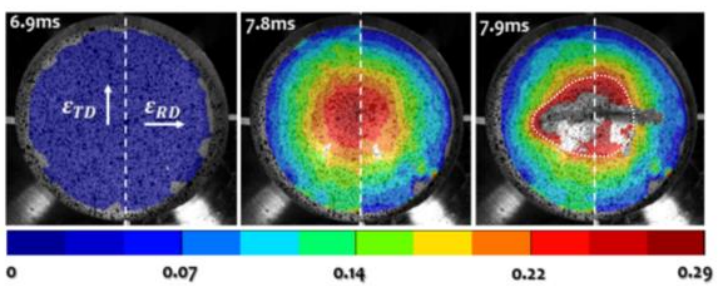

Fig. 4. Full-field contour plots of strain in the rolling and transverse directions, i.e., RD and TD resp., at different instants of the experiment at $16.5 \mathrm{~m} / \mathrm{s}$

The difference of the two strain fields gives a clear indication of the material anisotropy. The strain rate is calculated as time derivative of the through thickness strain given by eq. (2). The strain rate at the sample apex is presented as a function of time in Fig. 5. For the lowest velocity experiment, during plastic deformation, a quasi-constant strain rate ranging between $200-250 \mathrm{~s}^{-1}$ is obtained. After a stable plateau, the strain rate decreases due to the end of the experiment. For the highest velocity test, a stable strain rate of about $300-350 \mathrm{~s}^{-1}$ is imposed to the material. In this case, after the plateau, the strain rate increases exponentially, until the sample fracture. The exponential increment of strain rate, in the experiment at highest velocity, suggests strain concentration around the apex preceding the sample fracture.

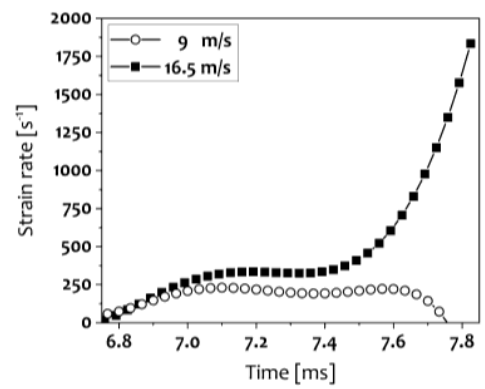

Fig. 5. Strain rate evolution with respect to the time for two different impactor velocities

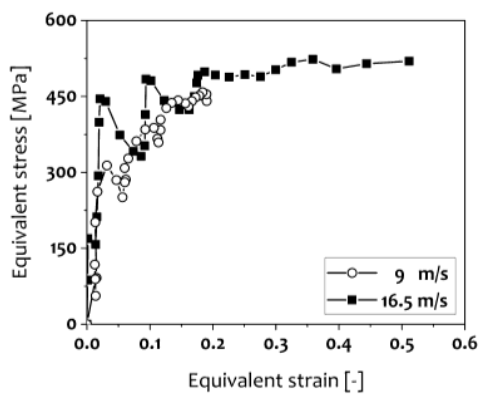

Fig. 6. Equivalent stress-strain curve obtained via high-speed bulge setup for two different impactor velocities

In Fig. 6, the equivalent stress of Eq. (1) is plotted with respect to the equivalent strain of Eq. (2) for both the impactor velocities. The strain gage recordings on the output bar allow to measure the bulge pressure using Eq (4), while the actual thickness and radius of curvature are quantified via stereo DIC measurements. Noticeable oscillations are observed in the stress level, especially during the initial stage of the experiment. Since the bulge pressure increases gradually without noticeable oscillations, the origin of the oscillation was investigated making used of the DIC data. Further analysis has revealed that during the initial stage of the experiment the sample starts vibrating. The ringing of the sample has a direct impact on the radius of curvature, especially during the first stage of the experiment. Moreover, the sample vibration increases with the test velocity. Indeed, the oscillations in the stress level observed for the experiment at $9 \mathrm{~m} / \mathrm{s}$ are almost negligible compared to these observed at $16.5 \mathrm{~m} / \mathrm{s}$. 


\section{Conclusions}

In this work a novel split Hopkinson bar bulge facility is presented. The special configuration of the Hopkinson bars leaves the sample surface fully accessible for high-speed camera monitoring during the entire experiment. The design of the hydraulic chamber and loading system leads to a nearly oscillation-free transmitted wave, which guarantees a steady sample loading. The strain fields in the rolling and transverse directions exhibit an elliptical distribution during the last stage of the experiment, suggesting the development of the material anisotropy with the sample deformation. A quasi-constant strain rate is imposed to the material around the apex for both test conditions. However, an exponential increment of the strain rate is observed for the experiment at the highest velocity, suggesting further concentration of the deformation before fracture. The flow curves obtained for quasi-biaxial loading and high strain rates are influenced by the sample vibration. The sample ringing mainly affects the initial stage of the sample deformation. Higher oscillations in the stress measurements are observed for the experiment performed at the highest velocity. It is important to highlight that the flow curves obtained via bulge tests cover a much wider strain range compared to tensile curves and are therefore highly valuable for forming. Additionally, the experimental campaign revealed the potential of the setup of being employed as a valid dynamic counterpart of the quasi-static bulge experiment.

The authors gratefully acknowledge the Research Foundation Flanders-FWO, application number $1 \mathrm{SC} 5619 \mathrm{~N}$. The authors thank the Ghent University technical staff Wouter Ost, Luc Van den Broecke and Pascal Baele for their valuable support on the realization of the setup.

\section{References}

[1] P. Verleysen, J. Peirs, J. Van Slycken, K. Faes, and L. Duchene, "Effect of strain rate on the forming behaviour of sheet metals," Journal of Materials Processing Technology, vol. 211, no. 8, Art. no. 8, (2011)

[2] T. Altan and A. E. Tekkaya, Eds., Sheet metal forming. Materials Park, Oh: ASM International, (2012)

[3] V. Grolleau, G. Gary, and D. Mohr, "Biaxial testing of sheet materials at high strain rates using viscoelastic bars," Experimental Mechanics, vol. 48, no. 3, pp. 293-306, (2008)

[4] M. Ramezani and Z. M. Ripin, "Combined experimental and numerical analysis of bulge test at high strain rates using split Hopkinson pressure bar apparatus," Journal of Materials Processing Technology, vol. 210, no. 8, pp. 1061-1069, (2010)

[5] R. Hill, "C. A theory of the plastic bulging of a metal diaphragm by lateral pressure," The London, Edinburgh, and Dublin Philosophical Magazine and Journal of Science, vol. 41, no. 322, Art. no. 322, (1950)

[6] W. Chen and B. Song, Split Hopkinson (Kolsky) Bar. Boston, MA: Springer US, (2011) 